\title{
Diagnosis and surgical treatment of arachnoid web
}

\author{
Ga-On Park ${ }^{1}$, Do-Yeon Kim², Hyun-Jun Jang ${ }^{1}$, Kyung-Hyun Kim ${ }^{1}$, Jeong-Yoon Park ${ }^{1}$, Dong-Kyu Chin ${ }^{1}$, Keun-Su Kim ${ }^{1}$, \\ Yong-Eun $\mathrm{Cho}^{1}$ \\ ${ }^{1}$ Department of Neurosurgery, Spine and Spinal Cord Institute, Gangnam Severance Hospital, Yonsei University College of Medicine, Seoul, Korea \\ ${ }^{2}$ Department of Neurosurgery, Kosin University Gospel Hospital, Kosin University College of Medicine, Busan, Korea
}

Arachnoid web is a rare disease entity that can cause progressive myelopathy and most often develops at the upper thoracic level. Its pathophysiology is unclear, but may be associated with degeneration of the septum posticum in the dorsal subarachnoid space, which alters the flow of cerebrospinal fluid (CSF) and subsequently leads to cord compression and syringomyelia. It often presents with pain, paresthesia, and extremity weakness. Arachnoid web is diagnosed by a typical pattern of displacement of the spinal cord, known as the scalpel sign, with intact ventral dura mater and disturbed but conserved CSF flow. Arachnoid web should be differentiated from other disease entities sharing the feature of ventral displacement of the dorsal spinal cord, such as arachnoid cyst or spinal cord herniation. The treatment for arachnoid web is surgical resection. We report a 66-year-old female who was diagnosed with arachnoid web in the dorsum of the spinal cord at the T3 level. She had suffered from weakness of both legs for 3 months. She underwent laminectomy of T3 and T4 and the dura was opened. The web was resected and the displacement of the spinal cord then improved.

Keywords: Arachnoid; Cerebrospinal fluid; Spinal cord compression; Diagnosis; Surgical procedures, operative

\section{Introduction}

The term "arachnoid web" was first mentioned by Mallucci et al. [1] in 1997 as one of the possible causes of idiopathic syringomyelia. They reported that arachnoid webs or pouches create syringomyelia by partially blocking the flow of cerebrospinal fluid (CSF) and can originate from the septum posticum. Then, the disease entity of arachnoid web was verified by Paramore [2] in 2000. He reported two cases of arachnoid web characterized by focal indentation of the dorsal thoracic cord that was not true arachnoid cyst but blocking CSF flow in magnetic resonance imaging (MRI) and computed tomography (CT) myelogram. Both patients presented with weakness of lower extremities and were treated with surgical resection, which produced improvement clinically and radiologically. Since then, a few case reports and studies have been pub-

Received: April 19, $2021 \quad$ Accepted: May 18, 2021

Corresponding Author: Yong-Eun Cho, MD, PhD

Department of Neurosurgery, Spine and Spinal Cord Institute, Gangnam

Severance Hospital, Yonsei University College of Medicine, Seoul 06273, Korea

Tel: +82-2-2019-3393; Fax: +82-2-3461-9229; E-mail: YECH0@yuhs.ac lished. Here, we introduce a case of a 66-year-old female who was diagnosed with arachnoid web in the spinal cord at the T3 level and was treated with surgical resection. To the best of our knowledge, 64 cases of arachnoid web have been reported, including our case.

\section{Case Report}

A 66-year-old female presented with slowly progressive motor weakness of both legs that had been aggravated for previous 3 months. She felt heavy-legged and ambulated with the help of a walker. She denied past history of spinal surgery or trauma. Physical examination showed weakness of both legs with Medical Research Council (MRC) grade 4, intact sensory function, intact bladder and bowel function, and negative upper motor neuron signs. Electromyography displayed myelopathy with prolonged tibial somatosensory evoked potential. Ventral displacement of the thoracic spinal cord at the T3 level was found on spine MRI and is known as the "scalpel sign" (Fig. 1). Ventral dura mater was intact, as was the CSF space at the ventral side of the spinal cord. There were no findings that suggested dural defect or spinal cord herniation and were no cysts, cystic walls, or mass-like lesions in the CSF 

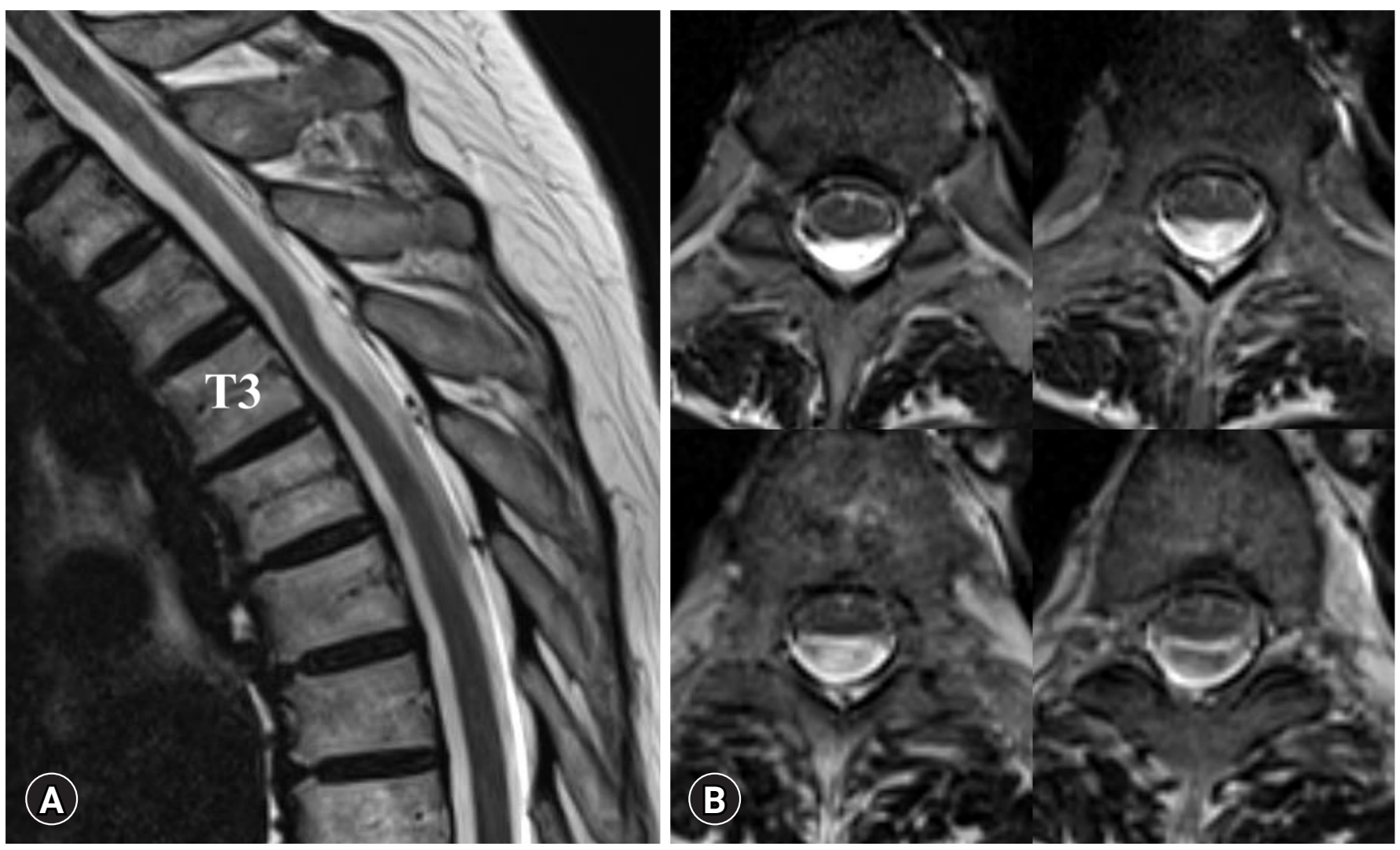

Fig. 1. Arachnoid web on preoperative T2-weighted image of magnetic resonance imaging. (A) A sagittal view. Ventral displacement and dorsal indentation of the spinal cord are seen at T3. A typical scalpel sign is observed. On the ventral side of the spinal cord, both the dura mater and the flow of cerebrospinal fluid (CSF) are intact. (B) Serial axial views. Ventral displacement of the spinal cord is seen, and the flow-void sign of the CSF is observed on the dorsal side of the spinal cord.

space. There were no findings of spinal cord signal change or syringomyelia. As a further study, CT myelography was performed and showed no filling defect that suggested complete blockage of CSF flow (Fig. 2). We performed open surgical exploration with the possibility of arachnoid web in mind. A midline vertical skin incision was made on the posterior upper back area with the patient in prone position. After total laminectomy of T3 and subtotal laminectomy of upper $\mathrm{T} 4$, some epidural granulation tissue was observed. We incised the dura mater and punctured the arachnoid mater, resulting in high-pressure release of CSF. Arachnoid membranes and adhesion bands covering the spinal cord were observed, dissected from the cord, and removed with microscissors (Fig. 3). Cord compression resolved after these procedures. Duroplasty was performed, and the wound was closed layer by layer. After the surgery, ventral displacement and dorsal compression of the spinal cord recovered radiologically on the postoperative MRI (Fig. 4), and the symptoms also improved.

Due to its nature of case report, the study was exempt from requiring informed consent from the participants.

\section{Discussion}

Arachnoid web is a rare disease that occurs mainly in the upper thoracic level with ventral cord displacement [3]. Pathophysiology of arachnoid web is not well-known, but it might be associated with blockage of CSF flow, formation of syringomyelia, and incomplete arachnoid cyst formation [4]. Arachnoid web should be differentiated from ventral cord herniation and arachnoid cyst [5] and can be diagnosed radiologically by the characteristic feature scalpel sign [6]. It usually manifests as symptoms of progressive myelopathy and can be treated successfully with surgical removal $[3,7]$.

Common symptom presentations are lower-extremity weakness, sensory loss, and bladder or bowel incontinence according to one systematic review of 19 articles and 41 patients [3]. Neuropathic back pain and myelopathy such as hyperreflexia, spastic paraparesis, clonus, and gait disturbance also have been reported [8]. Several predisposing factors are prior traumatic event or spine surgery, which can induce hemorrhage, infection, inflammation, or adhesion in the subarachnoid space. In a previous study, 5 of 14 

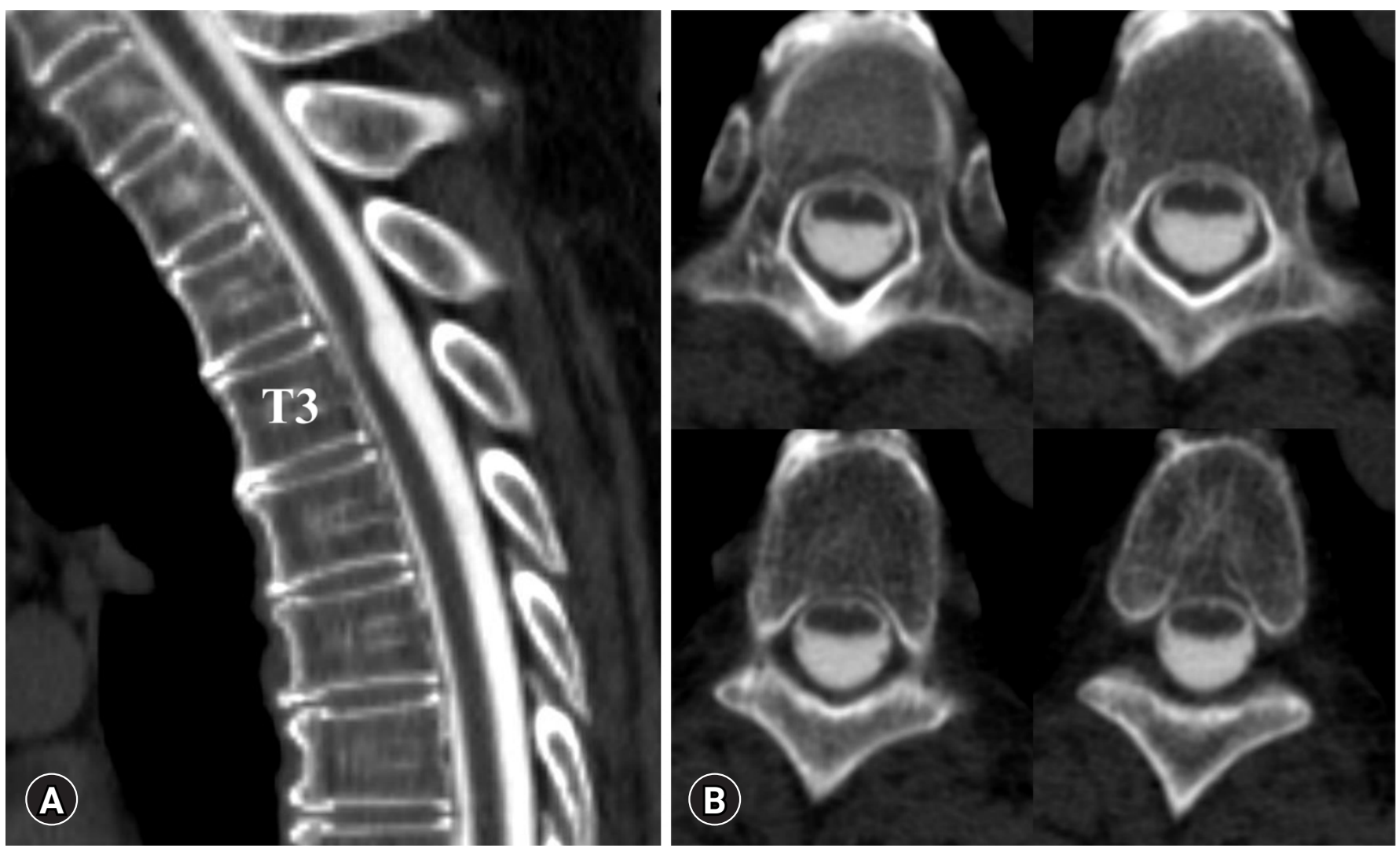

Fig. 2. Arachnoid web on preoperative computed tomography (CT) myelography. Contrast dye was injected by lumbar puncture at L2-3, and CT myelography was performed. (A) A sagittal view. As in magnetic resonance imaging, a typical scalpel sign is shown at the T3 level. (B) Serial axial views. Contrast dye fills all space of the cerebrospinal fluid (CSF), and any findings of filling defect suggesting complete blockage of the flow of CSF are not seen.

arachnoid web patients had history of trauma or spine surgery [9]. In our case, though the patient reported no history of spinal surgery or trauma, epidural granulation tissue was observed intra-operatively, suggesting unrecognized trauma.

The pathophysiology of arachnoid web is unclear. Anatomically, the spinal cord is floating in the CSF space and stably fixed to the meninges by ligaments. The paired denticulate ligaments connect pia mater to dura mater at both sides of the spinal cord. The septum posticum connects pia mater to dura mater at the dorsal side of the spinal cord. Arachnoid web is thought to originate from disruption of the septum posticum $[1,4]$. At the thoracic spine level, the septum posticum is important to stabilize the dorsal spinal cord against the ventral displacement of normal thoracic kyphosis [4]. This could explain the predilection of arachnoid web at the upper thoracic levels.

Syringomyelia often accompanies arachnoid web, which suggests that CSF flow is being disturbed. In one study, arachnoid web was related to syringomyelia in 12 of 14 case reports [9]. The term arachnoid web was referred to initially in a study of the causes of idiopathic syringomyelia [1]. In the study, preoperative myelogra- phy of arachnoid web showed partial blockage of CSF flow. Disturbed CSF flow due to arachnoid web causes abnormal accumulation of CSF, and the relatively high regional pressure results in spinal cord compression and displacement observed as the scalpel sign. Moreover, this intermedullary CSF pressure gradient can result in syringomyelia. The typical location of syringomyelia is either rostral or caudal to the arachnid web because the specific structure of arachnoid web can promote one-way flow of CSF and unidirectional syringomyelia [7].

Diagnosis of arachnoid web is based on the scalpel sign. This typical radiologic finding was first reported by Reardon et al. [6] in 2013. Its name originates from the shape of cord displacement resembling the surgical instrument scalpel. The radiologic findings of arachnoid web, arachnoid cyst, spinal cord herniation, and arachnoid adhesion are similar, and these disease entities might share pathophysiology. Chellathurai et al. [4] reported the disease spectrum of ventral displacement of the dorsal spinal cord and summarized the characteristics of these disease entities as follows: arachnoid web, preserved but disturbed CSF flow; arachnoid cyst, fluid-filled mass; spinal cord herniation, ventral dural defect; and 

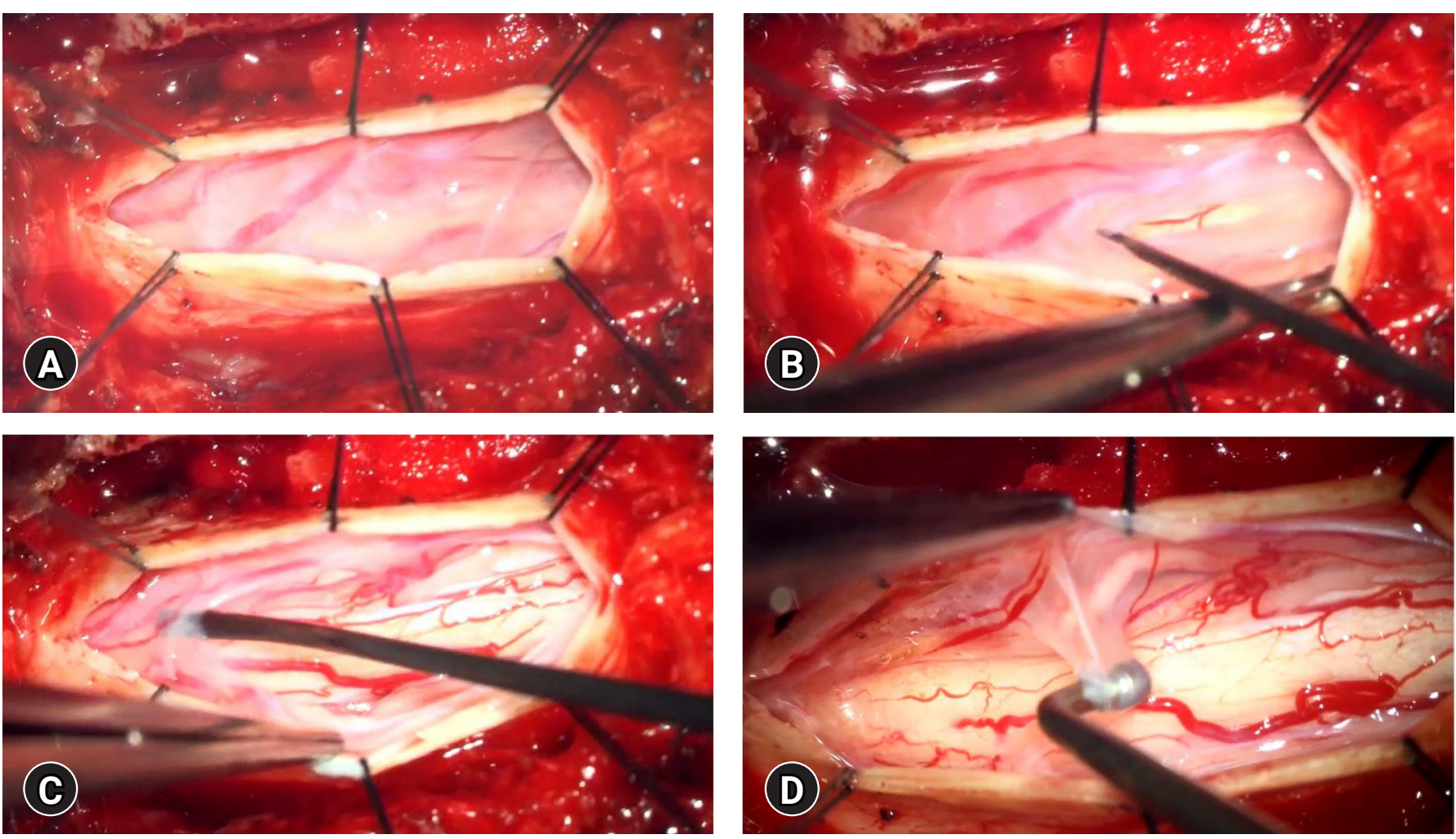

Fig. 3. Intraoperative findings of arachnoid web. Total laminectomy of T3 and subtotal laminectomy of upper T4 was performed. (A) After the dura mater was opened, the arachnoid web was identified as translucent tissues covering the spinal cord. As the arachnoid membrane punctured, the cerebrospinal fluid was released with relatively high pressure. (B) The arachnoid web was adhesive and band-like. (C) The arachnoid web was removed carefully, and the spinal cord was exposed. (D) Cord compression resolved after removing most of the arachnoid web.

arachnoid adhesion, less-severe form of ventral dural defect. Understanding these diseases elucidates differential diagnosis. Schultz et al. [5] reported that scalpel-shaped cord displacement and preservation of the ventral CSF plane suggest arachnoid web, while $\mathrm{C}$-shaped cord displacement and interruption of the ventral CSF plane suggest cord herniation. Hirai et al. [10] also reported differential diagnosis of arachnoid cyst, arachnoid web, and cord herniation. Spinal cord herniation involves a ventral dural defect and complete blockage of CSF flow at the ventral side of the cord. Arachnoid cyst usually shows cystic wall formation on MRI and disturbance of CSF flow on CT myelogram. Arachnoid web shows typical scalpel-shaped ventral cord displacement with preserved ventral CSF flow and dura mater, no visible cystic wall formation, and normal CSF filling on CT myelogram. Other reported diagnostic modalities include heavily $\mathrm{T} 2$-weighted constructive interference in steady state (CISS) sequence MRI and cine MRI. CISS sequence MRI can visualize the arachnoid web directly, in comparison to MRI or CT myelogram [11]. Cine MRI can quantitatively measure and visualize CSF flow [12]. Thus, arachnoid web is diagnosed by multi-modality imaging including MRI, CT myelogram, and cardiac-gated cine phase contrast MRI [13]. In our case, ventral displacement of the spinal cord, the scalpel sign, was observed on MRI and suggested possible diagnoses of spinal cord herniation, arachnoid cyst, and arachnoid web. Spinal cord herniation was ruled out by findings of intact dura mater and intact ventral CSF space on MRI. In addition, CT myelography ruled out arachnoid cyst based on absence of complete blockage of CSF flow and cystic mass formation. Thus, arachnoid web was diagnosed on MRI and CT myelogram.

Surgical resection is the gold standard treatment of arachnoid web. Most reported studies show improvement of symptoms and good prognosis after surgical resection of arachnoid web. Open laminectomy and resection of arachnoid web are widely used surgical methods $[1-3,6,10,14,15]$. Extent of resection need to be minimized to avoid secondary iatrogenic arachnoid adhesion that can aggravate symptoms even after surgical resection of arachnoid web. To prevent iatrogenic arachnoid adhesion, intraoperative dye injection into the subarachnoid lesion can be used. The dye delineates the extent of the lesion and to aid in determination of the resection margin [16]. Minimally invasive resection of arachnoid 

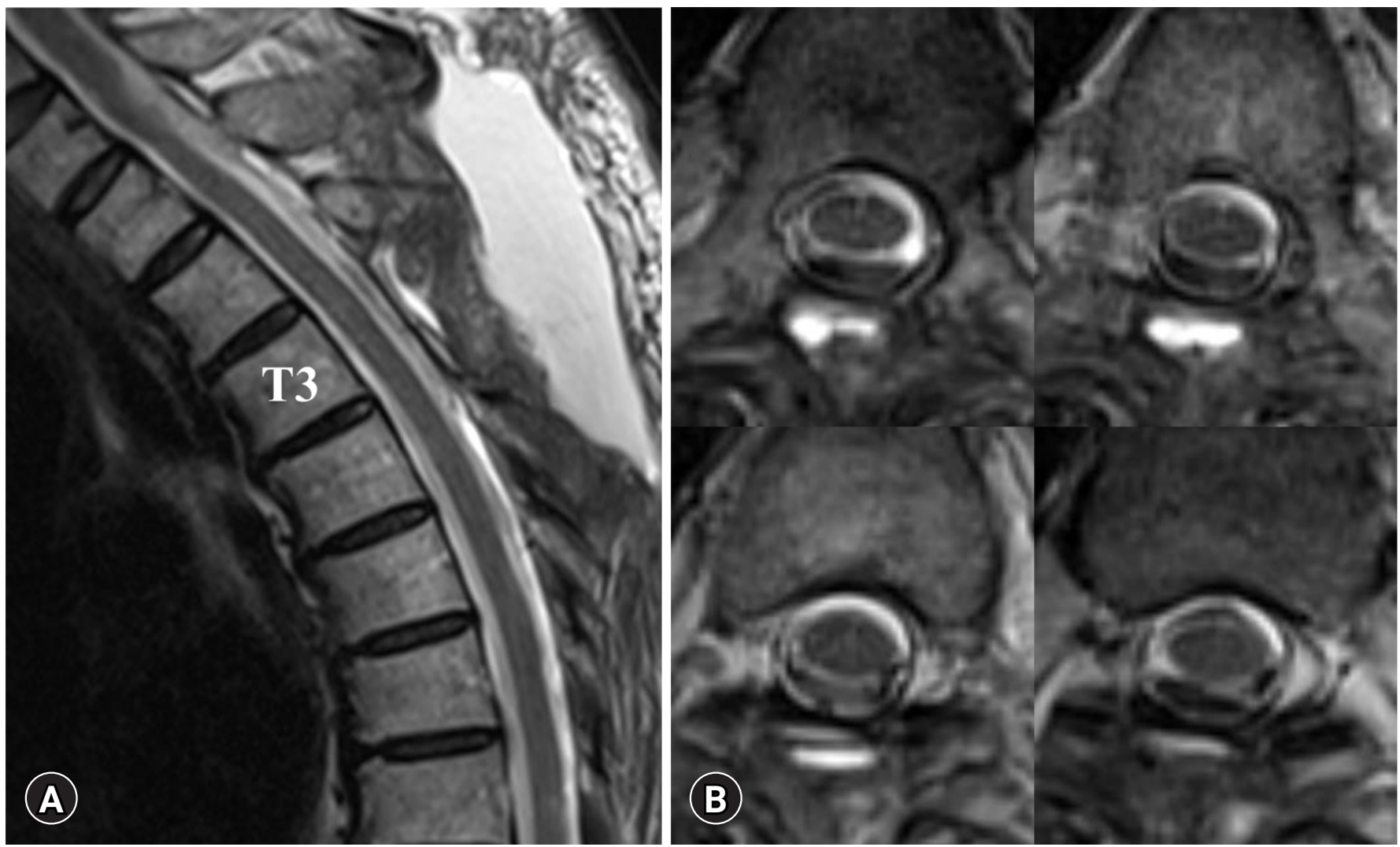

Fig. 4. Postoperative T2-weighted image of magnetic resonance imaging. (A) A sagittal view. Arachnoid web was removed and the scalpel sign is no longer visible. (B) Serial axial views. Ventral displacement and dorsal compression of the spinal cord at T3 resolved completely.

web was reported in two cases [17], and percutaneous intrathecal catheterization of arachnoid web was reported in one case [18]. Another case report presented a subarachnoid-subarachnoid shunt procedure for treatment of arachnoid web [19]. The patient in our case report received open surgical resection that resulted in improvement.

\section{Conclusion}

Arachnoid web is a rare disease but should be discerned from other similar pathologies of arachnoid cyst or spinal cord herniation. The key finding of arachnoid web is the scalpel sign on MRI, and differential diagnosis can be accomplished by various modalities such as spine CT myelogram, special MRI sequence of CISS, or cine MRI. Treatment for arachnoid web is surgical intervention including open resection, minimally invasive technique, and percutaneous catheterization.

\section{Conflicts of interest}

No potential conflict of interest relevant to this article was reported.

\section{ORCID}

Ga-On Park, https://orcid.org/0000-0003-3593-5458

Do-Yeon Kim, https://orcid.org/0000-0001-7750-925X

Hyun-Jun Jang, https:// orcid.org/0000-0001-6063-3510

Kyung-Hyun Kim, https://orcid.org/0000-0002-1338-5523

Jeong-Yoon Park, https://orcid.org/0000-0002-3728-7784

Dong-Kyu Chin, https://orcid.org/0000-0002-9835-9294

Keun-Su Kim, https://orcid.org/0000-0002-3384-5638

Yong-Eun Cho, https://orcid.org/0000-0001-9815-2720

\section{REFERENCES}

1. Mallucci CL, Stacey RJ, Miles JB, Williams B. Idiopathic syringomyelia and the importance of occult arachnoid webs, pouches 
and cysts. Br J Neurosurg 1997;11:306-9.

2. Paramore CG. Dorsal arachnoid web with spinal cord compression: variant of an arachnoid cyst? Report of two cases. J Neurosurg 2000;93(2 Suppl):287-90.

3. Nisson PL, Hussain I, Hartl R, Kim S, Baaj AA. Arachnoid web of the spine: a systematic literature review. J Neurosurg Spine 2019;31:175-84.

4. Chellathurai A, Balasubramaniam S, Gnanasihamani S, Ramasamy S, Durairajan J. Pathophysiology and grading of the ventral displacement of dorsal spinal cord spectrum. Asian Spine J 2018;12:224-31.

5. Schultz R Jr, Steven A, Wessell A, et al. Differentiation of idiopathic spinal cord herniation from dorsal arachnoid webs on MRI and CT myelography. J Neurosurg Spine 2017;26:754-9.

6. Reardon MA, Raghavan P, Carpenter-Bailey K, et al. Dorsal thoracic arachnoid web and the "scalpel sign": a distinct clinical-radiologic entity. AJNR Am J Neuroradiol 2013;34:1104-10.

7. Inoue J, Miyakoshi N, Hongo M, et al. Diagnosis and surgical treatment of thoracic dorsal arachnoid web: a report of two cases. Case Rep Orthop 2020;2020:8816598.

8. Ben Ali H, Hamilton P, Zygmunt S, Yakoub KM. Spinal arachnoid web-a review article. J Spine Surg 2018;4:446-50.

9. Zhang D, Papavassiliou E. Spinal intradural arachnoid webs causing spinal cord compression with inconclusive preoperative imaging: a report of 3 cases and a review of the literature. World Neurosurg 2017;99:251-8.

10. Hirai T, Taniyama T, Yoshii T, et al. Clinical outcomes of surgical treatment for arachnoid web: a case series. Spine Surg Relat Res 2019;3:43-8.
11. Grewal SS, Pirris SM, Vibhute PG, Gupta V. Identification of arachnoid web with a relatively novel magnetic resonance imaging technique. Spine J 2015;15:554-5.

12. Chang HS, Nagai A, Oya S, Matsui T. Dorsal spinal arachnoid web diagnosed with the quantitative measurement of cerebrospinal fluid flow on magnetic resonance imaging. J Neurosurg Spine 2014;20:227-33.

13. Nada A, Mahdi E, Mahmoud E, Cousins J, Ahsan H, Leiva-Salinas C. Multi-modality imaging evaluation of the dorsal arachnoid web. Neuroradiol J 2020;33:508-16.

14. Sridharan A, Heilman CB. Transverse dorsal arachnoid web and syringomyelia: case report. Neurosurgery 2009;65:E216-7.

15. Brodbelt AR, Stoodley MA. Syringomyelia and the arachnoid web. Acta Neurochir (Wien) 2003;145:707-11.

16. Yamaguchi S, Hida K, Takeda M, et al. Visualization of regional cerebrospinal fluid flow with a dye injection technique in focal arachnoid pathologies. J Neurosurg Spine 2015;22:554-7.

17. Vergara P, Barone DG. Minimally invasive excision of thoracic arachnoid web. World Neurosurg 2018;109:e81-7.

18. Qureshi AI, Bains NK, Balasetti VK, et al. percutaneous fenestration of a spinal arachnoid web using an intrathecal catheter: effect on cerebrospinal fluid flow and clinical status. World Neurosurg 2020;142:17-23.

19. Fujiwara Y, Manabe H, Izumi B, Shima T, Adachi N. microscope and fiberscope-assisted subarachnoid-subarachnoid (S-S) bypass: a novel surgical technique to reestablish cerebrospinal fluid flow in treating dorsal spinal arachnoid webs, diagnosed by cineMRI. Clin Spine Surg 2018;31:58-64. 